\title{
Criminologie
}

\section{Le processus décisionnel de libération conditionnelle}

\section{Maurice Gauthier}

Volume 14, numéro 2, 1981

Libération conditionnelle : évolution et application (1899-1981)

URI : https://id.erudit.org/iderudit/017140ar

DOI : https://doi.org/10.7202/017140ar

Aller au sommaire du numéro

Éditeur(s)

Les Presses de l'Université de Montréal

ISSN

0316-0041 (imprimé)

1492-1367 (numérique)

Découvrir la revue

Citer cet article

Gauthier, M. (1981). Le processus décisionnel de libération conditionnelle. Criminologie, 14(2), 61-72. https://doi.org/10.7202/017140ar d'utilisation que vous pouvez consulter en ligne.

https://apropos.erudit.org/fr/usagers/politique-dutilisation/ 


\section{LE PROCESSUS DÉCISIONNEL DE LIBÉRATION CONDITIONNELLE \\ Maurice Gauthier}

\section{LE PROBLÈME}

Abondante, la littérature sur le processus décisionnel en matière de libération conditionnelle remonte à plusieurs décennies. Si les travaux des plus récentes années révèlent que des avenues et hypothèses intéressantes ont été explorées, notamment sur la prédiction et l'évaluation du comportement du détenu, ils indiquent surtout que l'on s'interroge encore non seulement sur la valeur réelle des techniques utilisées mais sur la possibilité même d'en arriver à une prise de décision rationnelle et objective en matière de libération conditionnelle*.

Nous comprenons que des critiques sévères puissent être dirigées contre cette mesure surtout lorsqu'elle est associée à des crimes violents ou crapuleux, mais doit-on oublier pour autant qu'elle présente un taux de succès fort respectable dans tous les pays où elle est en application?

Selon les plus récentes études effectuées au Canada, ce n'est pas tant la marge d'erreurs mais plutôt le processus décisionnel même en matière de libération conditionnelle qui est remis en cause.

Ainsi, en 1976, la Commission de réforme du droit, dans son rapport sur Le Processus de libération conditionnelle (6), insiste sur la nécessité d'un ensemble de critères clairs, cohérents et explicites pouvant servir de base aux décisions en matière de libération conditionnelle (p. 147).

En 1977, le sous-comité perlementaire, présidé par monsieur MacGuigan (7), précise que :

Les problèmes sont dus à l'imprécision des critères sur lesquels on se fonde pour accorder ou refuser une libération conditionnelle, à la multiplicité des sources de renseignements et enfin, à l'absence de mécanismes d'évaluation appropriés d'une décision essentiellement fondée sur le jugement subjectif des commissaires (paragraphe 745 ).

Des critiques et des remarques de ce genre ne sont pas nécessairement nouvelles; mais compte tenu de leur intensité et de

* Pour détails voir les ouvrages (1), (2), (3), (4), (5) et (6), cités dans la bibliographie. 
leur crédibilité accrues, il me semble opportun d'ajouter le point de vue d'un décideur sur le sujet.

Il apparaît d'abord important de définir le cadre juridictionnel étroit à l'intérieur duquel ce processus décisionnel s"inscrit, de considérer les techniques inhérentes à ce processus et d'en analyser les forces et les faiblesses.

Nous examinerons par la suite les interrelations qui existent entre les divers secteurs de l'administration de la justice et la mesure de libération conditionnelle ce qui permettra de faire ressortir la complexité et les nuances du processus décisionnel même.

LA LIBÉRATION CONDITIONNELLE : SON CADRE JURIDICTIONNEL Comme le rappelle Létourneau (8) :

La libération conditionnelle est une mesure de réinsertion sociale par laquelle un détenu, sous certaines conditions et sous surveillance, est autorisé par une commission de libération à purger sa peine d'emprisonnement dans la conmunauté plutôt qu'en milieu carcéral. Elle intervient donc après un jugement de culpabilité ordonnant une détention (p. 564).

La libération conditionnelle n'est donc pas une sentence, mais une décision prise par un organisme quasi judiciaire qui permet de réévaluer les modalités d'application d'une sentence d'emprisonnement déjà prononcée par un tribunal.

Les principaux pouvoirs de la Commission québécoise ainsi que les règles d'admissibilité à son programme sont clairement spécifiés dans des textes de loi ou de règlements adaptés au contexte constitutionnel canadien (9) (10) (11). Il y est notamment stipulé que la libération conditionnelle ne saurait être octroyée lorsqu'il y a un risque sérieux que la société n'en subisse un préjudice grave ou que le bénéficiaire ne se conforme pas aux conditions, généralement incorporées à sa libération.

C'est à ce niveau que certains parlent du paradoxe de la libération conditionnelle. D'une part, le juge impose la sentence dans la légalité et, d'autre part, toujours dans la légalité, une commission de libération conditionnelle peut décider d'une application différente de la sentence. Les concepts de punition suite à une offense commise et de réinsertion sociale s'y retrouvent ainsi côte à côte. Le prétexte à la critique sur l'efficacité de la mesure s'en trouve d'autant plus alimenté si une récidive en cours de surveillance survient, quel que soit le contexte où la décision initiale a été prise. 
REVUE DES PRINCIPALES TEChNIQUES UTILISÉES DANS LE PROCESSUS DÉCISIONNEL

Pour les fins du présent article, je m'en tiendrai à l'analyse des trois techniques les plus en usage dans les diverses juridictions où la mesure de libération conditionnelle est en application. Ce sont :

1) les tables de prédiction statistique;

2) les « guidelines " américains;

$3)$ les critères décisionnels.

\section{Les tables de prédiction statistique}

C'est au cours des années 1920 que furent utilisées pour la première fois aux États-Unis les tables de prédiction. Celles-ci, construites à partir de comparaisons entre groupes de détenus possédant des caractéristiques similaires, servent à évaluer les probabilités à l'égard du comportement futur d'un détenu. Elles ne prédisent pas sa récidive ou sa non-récidive; elles ne font qu'indiquer qu'il appartient à un groupe qui, comme tel, possède une probabilité élevée, moyenne ou basse de respecter les conditions d'une libération conditionnelle.

L'Angleterre en particulier utilise les tables de prédiction statistique comme instrument de base pour évaluer les risques de récidive d'un détenu et déterminer les cas devant être soumis à la Commission des libérations conditionnelles (13).

Dans ce pays, bien qu'on reconnaisse le caractère utilitaire de cette technique, on n'en fait pas moins ressortir deux faiblesses importantes : d'abord la nécessité de procéder à une révision constante des tables, puisque les données s'appuient sur le passé, c'est-à-dire sur une population qui à un moment donné présentait des caractéristiques similaires; de plus, les tables ne tiennent pas compte des différences qualitatives que l'on retrouve d'un établissement à l'autre tant du point de vue des programmes, du personnel, que de l'aménagement. Il s'agit en somme d'un instrument d'information indicatif de certaines tendances, dont la fugacité même renforcit d'autant l'importance de la discrétion, du jugement et de l'expérience individuelle dans le processus décisionnel.

\section{Les « guidelines》 américains}

Aux États-Unis, on le sait, une tendance marquée en faveur d'une discrétion plus structurée s'est développée au cours des dernières années, principalement sous la forme de "guidelines » (14) (15) dont la flexibilité souhaitée se situerait entre la discré- 
tion la plus absolue et l'application rigide de règles fixes et mécaniques, mais, "Discretion should be structured and visible rather than eliminated or controlled externally by the system , explique le ministère de la Justice des États-Unis (16), qui rappelle ainsi que la discrétion demeure un principe fondamental de la mesure.

Toutefois, après avoir étudié les divers modèles et avoir rencontré des responsables américains en matière de libération conditionnelle, nous nous demandons si cette mesure ne constitue pas avant tout une réponse défensive aux critiques sévères dirigées contre le processus décisionnel et si son utilisation actuelle, empreinte de rigidité et d'automatisme, n'ouvre pas déjà la voie à l'informatisation du processus et à la réduction éventuelle, sinon à la disparition du pouvoir discrétionnaire lui-même.

Il est certes trop tôt pour apprécier toute la portée juridique et sociale de cette nouvelle technique mais compte tenu des nombreuses interrogations qu'elle suscite, elle devrait, à notre avis, faire l'objet d'études approfondies avant d'en préconiser ici l'application, notre cadre légal, nos dimensions socio-économiques ainsi que notre contexte culturel étant sensiblement différents de ceux des états américains où elle est déjà instaurée.

\section{Les critères décisionnels}

Dans des juridictions comme la nôtre qui n'ont recours ni aux tables de prédiction ni aux "guidelines», si la discrétion demeure importante, la nécessité d'établir la prise de décision sur des critères clairs, cohérents et explicites l'est tout autant pour éviter le genre de critiques que subissent les autres formules.

Cependant, avec la Commission de réforme du droit (6) nous croyons que :

Il suffit de lire quelques dossiers de détenus et d'observer quelques audiences pour se rendre compte que ces critères, quoique indispensables, ne sauraient éliminer le pouvoir d'appréciation discrétionnaire que doivent conserver les décideurs face à l'infinie diversité des situations personnelles et à l'évolution constante du milieu social (p. 128).

Ce que l'on conteste en somme, ce n'est pas le pouvoir discrétionnaire même, mais le fait que les décisions ne soient pas toujours limpides, adaptées aux situations individuelles ou traduites de manière à convaincre les détenus de leur bien-fondé et à informer clairement les divers intervenants des motifs décisionnels.

C'est d'ailleurs pourquoi le législateur québécois, conscient de ce problème, a indiqué dans la loi (10), à l'article 23 , les 
principaux éléments dont la Commission doit tenir compte dans l'octroi ou le refus d'une libération. Cet article se lit ainsi :

23. En rendant sa décision, la commission tient compte notamment de la personnalité et du comportement du détenu, de son habilité à remplir ses obligations, de ses projets, de ses relations familiales et sociales, de ses emplois antérieurs, de ses aptitudes au travail, de son casier judiciaire ou de sa conduite pendant une période d'absence temporaire accordée en vertu de la Loi sur la probation et sur les établissements de détention ou pendant une période de détention ou de libération conditionnelle.

Comme le souligne Létourneau (8) :

La liste n'est qu'indicative : les notions de réinsertion sociale, de risque, de préjudice grave à la société et d'obéissance aux conditions demeurent les critères fondamentaux d'octroi ou de refus (p. 574).

Létourneau, commentant plus à fond cet article de la loi (8), précise que, s'il ne change rien au droit existant en ce domaine, il a cependant l'avantage de cristalliser dans un texte des notions auparavant vagues et souvent mal connues des détenus. La définition claire de critères bien qu'elle soit importante et ajoute plus de crédibilité à la prise de décision, n'en constitue néanmoins qu'un palliatif et peut prêter flanc aux mêmes critiques que celles qui sont dirigées vers les tables de prédiction et les « guidelines».

Ce qui semble primordial, en effet, c'est certes une justification logique et motivée de la décision, mais aussi la conviction pour l'intéressé et pour les principaux intervenants que la bonne décision a été prise.

Au Québec, la réalisation de cet objectif est, à notre avis, particulièrement facilitée par les interrelations qui existent entre les divers secteurs de l'administration de la justice et la mesure de libération conditionnelle elle-même.

LIBÉRATION CONDITIONNELLE ET PROCESSUS JUDICIAIRE

La libération conditionnelle, rappelons-le, porte sur la réévaluation des modalités d'application d'une sentence d'emprisonnement déjà prononcée par un tribunal et fait partie intégrante du processus judiciaire au même titre que l'arrestation, le procès et l'incarcération.

L'examen, dans les paragraphes suivants, des principales caractéristiques des rapports étroits entre la mesure de libération conditionnelle avec les autres secteurs de la justice, permettra de 
bien situer le cadre et les limites d'intervention d'une Commission des libérations conditionnelles de même que la complexité inhérente au processus décisionnel. Ces caractéristiques sont :

\section{Complémentarité du rôle des intervenants}

En matière de justice pénale et criminelle un rôle précis et complémentaire est assigné à chacun des intervenants, et ce, à toutes les étapes du processus judiciaire.

Ainsi, il revient aux policiers de procéder à l'arrestation des contrevenants aux lois pénales et criminelles et aux enquêtes requises ou utiles au tribunal. Puis, au cours du procès, le tribunal statue sur la culpabilité ou l'innocence de l'inculpé et prononce, s'il y a lieu, la sentence. Si l'incarcération s'impose, il revient à des auxiliaires de la justice, soit aux membres du personnel des établissements de détention de voir à la bonne exécution des décisions du tribunal. La Commission des libérations conditionnelles intervient à son tour dans le cadre qui lui est défini par la loi en appuyant ses décisions et leur mise en application sur la callaboration et la consultation des mêmes auxiliaires, y compris ceux de la probation et toute autre personne susceptible d'apporter une contribution. C'est dans ce sens qu'il faut parier de la complémentarité du rôle des intervenants du système judiciaire. Même le détenu, par sa présence à l'audience où il peut exprimer son point de vue ou recourir à un représentant, participe aussi au processus (10).

2. Étape précise de l'intervention de la Commission des libérations conditionnelles dans le processus judiciaire.

La prise de décision survient à un moment précis du processus judiciaire et sur une personne déterminée. D'une part, les textes de loi spécifient les dates d'admissibilité à la libération conditionnelle et, d'autre part, la mesure s'applique à un détenu sentencé à une peine privative de liberté (9) (10). Elle s'effectue en regard d'un programme particulier et des possibilités présentes de succès ou d'insuccès du principal intéressé et selon des critères clairement définis dans la loi (9) (10).

Qu'à ce moment précis du processus, les décideurs puissent diverger d'opinion ou prendre une décision différente de celle de l'intervenant précédent ne constitue pas, à notre avis, un paradoxe, mais l'exercise normal d'une responsabilité prescrite par la loi. Ainsi, il arrive qu'un juge conclue, après procès, à l'acquittement d'un prévenu que les policiers, au moment de l'arrestation, avaient 
des raisons légitimes de croire coupable. Le même principe vaut, croyons-nous, pour les décisions en matière de libération conditionnelle.

Lorsque la mesure ne s'applique pas, le détenu, signalons-le, peut avoir accès à diverses autres possibilités qui relèvent de l'administrateur de l'établissement telles que : les programmes d'absence temporaire, le transfert à des centres spécialisés ou les programmes institutionnels de travail.

\section{Les aléas de la décision}

En raison des facteurs nombreux et complexes, tant psychologiques, familiaux, sociaux et autres, qui caractérisent la clientèle admissible à la libération conditionnelle, il nous semble illusoire d'escompter que quelque décision à cet égard puisse revêtir un caractère d'infaillibilité. Dans ce secteur, comme ailleurs, une décision se prend dans le temps et dans une conjoncture particulière. Tout au plus, pouvons-nous dans un premier temps chercher à justifier la nôtre à la lumière des données et des faits précis disponibles et mis en évidence, en veillant à réduire au minimum les risques d'erreur. $\mathrm{Si}$, par la suite, la conjoncture change et que l'incidence de faits nouveaux nécessite une réévaluation de la décision, il faut, bien sûr, réajuster le tir. C'est la méthode suivie dans le monde des affaires et c'est aussi celle de l'administration de la justice. En regard de la libération conditionnelle, la loi a d'ailleurs prévu un mécanisme de suspension et de révocation de la libération voire même un mécanisme de révision (10).

Certes, des règles de prédiction, des «guidelines 》 ou des critères définis peuvent constituer des balises opérationnelles utiles et même, dans une certaine mesure, contribuer à accroìtre la qualité des décisions, mais aussi sophistiqués qu'ils puissent être, ces outils ne sauront jamais prévoir les réactions d'un être humain dans ses agirs de demain, non plus que permettre une discrimination suffisante dans l'évaluation de ses antécédents ou de son comportement actuel.

\section{Indépendance de l'organisme de décision}

La décision en matière de libération conditionnelle vise d'abord à modifier le statut légal d'un justiciable, de détenu à libéré conditionnel et, ensuite, à le transférer de la responsabilité d'un directeur d'établissement de détention à celle d'un agent de surveillance. 
Pour en assurer la plus grande objectivité, il convient, à notre avis, que cette décision soit prise par un organisme jouissant d'une indépendance d'action analogue à celle d'un tribunal et qu'il y ait une nette distinction entre la prise de décision elle-même par lorganisme et son application par les auxiliaires de la justice.

Il s'agit pour nous d'un principe fondamental que l'on retrouve d'ailleurs dans l'ensemble des juridictions canadiennes comme dans celles des autres pays.

En Californie (17), par exemple, cette responsabilité relève d'un «Board of Prison Terms » alors qu'en France, il revient à un juge de l'application des peines de statuer sur la mesure de libération conditionnelle. En Angleterre, il existe, comme ici, une commission des libérations conditionnelles, mais le pouvoir exécutif constitue l'autorité décisionnelle pour une partie des cas. Au Québec, par contre, le pouvoir de la Commission est souverain et non subordonné au pouvoir exécutif.

Si, selon la façon dont on estime qu'elle peut le mieux répondre aux attentes de la société, la formule varie d'un pays à l'autre et la responsabilité n'en est pas toujours confiée à un organisme appelé "Commission de libération conditionnelle», on retiendra que nulle part la mesure comme telle n'a été abolic, mais remplacée par un mécanisme qui permet la libération surveillée et avant terme des personnes incarcérées.

\section{Individualisation des décisions}

Nous insistons enfin sur l'importance d'adapter les décisions aux situations individuelles des détenus. Dans des libres propos qu'il confiait au bulletin - Justice - en mars 1980 (18), le juge en chef Yves Mayrand, de la Cour des sessions de la paix, affirmait :

Et je suis un de ceux qui prétendent que l'uniformité des sentences conduit à plus d'injustice que l'individualité des sentences. Pensez-donc! Ce serait beaucoup plus facile pour les juges d'avoir une table de critères et de points, de disposer de données fixes. Aussi, et je le dis sans ambages, ceux qui prétendent qu'il faudrait uniformiser les sentences ne savent pas de quoi ils parlent. Il n'y a pas deux cas pareils.

Nous partageons, bien sûr, ce point de vue qui accorde une grande place au pouvoir discrétionnaire du juge ou d'une commission de libération conditionnelle, en prenant pour acquis que toute libération octroyée, quelles que soient les précautions dont 
la décision soit assortie, comporte des risques d'échec et puisse à l'occasion susciter des remous.

\section{CRÉDIBILITÉ DE LA DÉCISION}

L'étude des interrelations qui existent entre les divers secteurs du processus judiciaire aide, certes, à mieux situer le cadre et les limites de la mesure de libération conditionnelle de même que la complexité inhérente au processus décisionnel, mais, en donnant une large part à la discrétion, on ouvre inévitablement la porte aux critiques allégant le caractère arbitraire et subjectif des décisions. Comment résoudre ce problème? Comment, en effet, s'assurer de créer une atmosphère où les intervenants du processus judiciaire et la personne incarcérée partageront la conviction que la meilleure décision a été prise?

Le problème est de taille. Au Québec, au moment de la rédaction de la Loi sur la libération conditionnelle (10), nous avons pris conscience de la difficulté et avons cherché à y introduire les éléments qui permettraient d'y faire face. Pour y parvenir, nous avons accordé une grande importance à une participation active des principaux intervenants et du détenu à toutes les étapes de l'audience. Nous avons considéré essentiellement l'ouverture des audiences à toute personne directement intéressée, dont non seulement la présence mais aussi sa participation active est encouragée dans les limites de son implication dans la mesure.

S'impose aussi la nécessité d'une explication des motifs sur lesquels s'appuie la décision. D'où, dans la loi, la large part accordée aux droits de la personne incarcérée et aux modalités de sa participation à l'audience.

En tout premier lieu, le législateur est parti de la prémisse qu'il incombait au principal intéressé de prouver à la Commission son aptitude à bénéficier d'une libération conditionnelle, puisque c'est dans son cas qu'elle sera appliquée. Le fardeau de la preuve lui échoit donc. Dans un tel contexte, la personne incarcérée doit elle-même mettre au point un plan de sortie qu'elle devra exposer en détail devant la Commission. Elle se doit de réaliser que la libération conditionnelle est son affaire d'abord et avant tout. Sa participation active à l'audience s'avère ainsi essentielle.

Cette préoccupation, nous la retrouvons traduite dans les articles de la loi (10) relatifs non seulement à la préparation du détenu en vue de l'audience, mais aussi à sa présence devant la 
Commission et aux modalités selon lesquelles la décision doit lui être communiquée.

À l'article 16 de la loi sur la probation et sur les établissements de détention (12), il y est spécifié que l'administrateur de l'établissement doit l'informer des dispositions de la loi, ce qui se fait à la fois verbalement et par la remise de documents dont un guide détaillé d'informations sur la mesure. De plus, tel que stipulé à l'article 11 du règlement (11), il se doit d'être informé deux semaines à l'avance de l'heure et de la date de l'audience. Les précautions sont donc prises pour qu'en prévision de l'audience, la personne incarcérée ait en main l'information pertinente et surtout qu'elle sache dans le détail le rôle qu'elle est appelée à y jouer. C'est un droit que lui accorde la loi.

Pour l'audience, ainsi que le stipule l'article 32 de la Loi favorisant la libération conditionnelle des détenus $(10)$, le détenu a droit d'être présent et de se faire entendre devant la Commission. Bien plus, parce que le fardeau de la preuve repose sur lui, le deuxième alinéa du même article lui permet de se faire représenter devant la Commission par toute personne de son choix ou d'en être assisté, sauf par un détenu incarcéré dans un autre établissement de détention. Tout est donc prévu dans le cadre même de la loi pour faciliter de sa part la présentation adéquate de son point de vue.

Enfin, à l'article 33 de la même loi, nous lisons que la Commission doit rendre, avec diligence, une décision écrite et motivée. En pratique, la décision est communiquée le même jour verbalement au détenu et à son ou ses représentants. Que ce soit un refus ou un octroi, les motifs de la décision lui sont expliqués. La décision écrite qui lui est remise s'avère un bref résumé de ce qui s'est dit verbalement.

Si le texte de loi accorde une grande importance à la définition du rôle du détenu, il en est de même pour les intervenants les plus directement impliqués. Ainsi, à l'article 22 de la même loi, nous voyons qu'avant de rendre sa décision, la Commission doit consulter l'administrateur de l'établissement de détention où le détenu est incarcéré. Dans les faits, et tel que spécifié à l'article 13 du règlement (11), le représentant de l'administrateur assiste à l'audience. Souvent, l'agent de probation, ou un représentant qui, en vertu de l'article 12 de la Loi sur la probation et sur les 
établissements de détention (12), sera appelé à jouer le rôle de surveillance advenant l'octroi, est aussi présent.

De façon à rencontrer les deux objectifs auxquels doivent tendre tous les intervenants du processus judiciaire, il apparaît en effet important que, quelle que soit la décision prise, positive ou négative, elle ait pour but de protéger la société et de favoriser la réinsertion sociale du détenu. Advenant un refus, le représentant de l'établissement, de par sa présence, est immédiatement saisi des motifs et peut par la suite apporter au détenu le support adapté dont il aura besoin jusqu'à la fin de son incarcération et en préparation d'un retour éventuel au sein de la société. Si la réponse est positive, les explications favorisent chez l'agent de probation une meilleure compréhension de la situation particulière du futur libéré et contribuent, par la même occasion, à faciliter le déroulement normal de la surveillance.

L'article 22 de la loi (10) ajoute par ailleurs que la Commission peut consulter toute autre personne. De fait, l'une des conditions de la libération comportant le rapport à la police, le corps policier de l'endroit où réside le détenu est systématiquement contacté et consulté en vue d'obtenir son opinion. Il y est aussi informé des motifs de la décision. Sa contribution à la surveillance en fait un des intervenants privilégiés.

Comme on peut le constater, nous partageons l'opinion, au Québec, qu'une participation active des principaux intéressés, et plus particulièrement du détenu, aux diverses étapes de l'audience, constitue la meilleure protection contre les critiques généralement dirigées vers la mesure de libération. Notre façon de procéder permet, selon nous, à la Commission de jouir de la crédibilité dont elle a besoin dans ses prises de décision, le principal intéressé et les intervenants immédiats y participant à toutes les étapes du processus.

Nous ne prétendons certes pas que notre formule est sans faille, mais elle semble tout de même répondre aux préoccupations présentes des personnes incarcérées et des principaux intervenants puisque, depuis sa mise en opération le ler avril 1979, elle n'a pas créé de remous importants ou suscité de critiques sévères au Québec.

Pour nous donc, des règles de procédure bien définies, la possibilité pour les intervenants et le détenu de s'exprimer librement, le souci de procéder dans la dignité et le respect de tous et 
la manière même de rendre une décision sont autant de gages de sa qualité, de son acceptation et de la crédibilité morale même de l’organisme de décision.

\section{BIBLIOGRAPHIE}

1) GOTTFREDSON, M.D., HOFFMAN, P.B., SIGLER, M.H. et WILKINS L.T., Making Parole Policy Explicit - Crime and Delinquency, janvier 1975 .

2) HOFFMAN, P.B. et DE GOSTIN, L.K., Parole Decision - Making : Structuring Discretion, Federal Probation, décembre 1974.

3) WILKINS, L.T., A Typology of Decision-Makers? A Theoretical and Speculative Contribution : *Parole : Legal Issues/Decision Making Research» W.E., Amos et C.L. Newman, eds., New York, Federal Legal Publications, 1975, p. 159-168.

4) McCALL, C.C., The Future of Parole - In Rebuttal of $S .1437$, Federal Probation, vol. 42, $\mathrm{n}^{\circ} 4,1978$, p. 3-10.

5) TAYLOR, E.L., In Search of Equity : The Oregon Parole Matrix, Federal Probation, vol. $n^{\circ} 1,1979$, p. 52-59.

6) CARRIÈRE, P. et SILVERSTONE, S., Le Processus de libération conditionnelle, Commission de réforme du droit du Canada, 1976.

7) MACGUIGAN, M., Sous-comité sur le régime d'institutions pénitentiaires du Canada, Gouvernement du Canada, 1977.

8) LEtoURnEAU, G. La Commission québécoise des libérations conditionnelles, Revue du Barreau, tome 39, $\mathrm{n}^{\circ} 3$, mai-juin 1979.

9) Loi sur la libération conditionnelle des détenus, S.R.C. 1970, c. P.2. (avec modification), Règlement sur la libération conditionnelle de détenus (1978) 112 C.P. 1978-5028 (avec modification).

10) Loi favorisant la libération conditionnelle des détenus (L.R.Q., c. L-1.1.).

11) Règlement sur la libération conditionnelle des détenus, Décret 2790-80 du 3 septembre 1980, Gazette officielle du Québec, $112^{\mathrm{e}}$ année, $n^{\circ} 47$.

12) Loi sur la probation et sur les établissements de détenus (L.R.Q., c. P-26).

13) Parole in England and Wales - Chapter 2 -, Prediction for Parole, Home Office Research Study, $\mathrm{n}^{\circ} 38,1977$.

14) HOFFMAN, P.B., Fedcral Parole Guidelines s Three Years of Experience, United States Parole Commission, Research Unit, Report Ten.

15) BRODERICK, B.A. et COSGROVE, C.A., The Process of Adopting a System of Guidelines in New York State (Paper presented at the American Society of Criminology), november 1979.

16) National Institute of Law Enforcement and Criminal Justice : Classification for Parole Decision Policy, U.S. Department of Justice, juillet 78.

17) WAY, H., State of California Board of Prison Terms, Summary of Policies and Procedures, 1980.

18) MAYRAND, Yves, juge en chef, « Libres propos avec Yves Mayrand Bulletin Justice, mars-avril 1980. 